\title{
Atmospheric environmental monitoring and evaluation based on experimental statistical methods
}

\author{
Lihong Sun \\ The Basic Science College, Harbin University of Commerce, Harbin 150028, China
}

\begin{abstract}
With the gradual improvement of social economy, the ecological environment is gradually deteriorating. In order to obtain higher economic benefits, enterprises often sacrifice the natural environment. After entering the new era, the public pays more and more attention to the ecological environment, and the relevant state departments also begin to use a variety of scientific and technological means to monitor the environment in order to obtain accurate information, find pollution problems in time, and take effective measures to control them. This is of great significance to improve the problem of environmental pollution. In this paper, combined with the actual situation, the application and layout methods of atmospheric environmental monitoring are analyzed in detail, in order to provide reference and reference for the related work in the future. In this paper, it is pointed out that atmospheric environmental monitoring is the process of measuring the concentration, observation and analysis of the change of pollutants in the atmosphere and the shadow response of the environment. The experimental statistical method is the product of the digital age, and it has its own advantages for monitoring the atmospheric environment, such as climate assessment, environmental assessment, atmospheric disaster prevention and early warning, etc., all based on the application of the experimental statistical method. Taking the digital measurement as the cut-in point, this paper describes the object and appearance of atmospheric monitoring, and focuses on the application strategy of atmospheric environmental monitoring test statistics, in order to clarify the importance of digital measurement and provide reference.
\end{abstract}

Keywords: Atmospheric environmental monitoring, experimental statistical methods, monitoring and evaluation

\section{Introduction}

Digital measurement as an effective means of atmospheric environment monitoring, the use of digital instruments to measure the atmospheric environment, has a good human-computer interaction function, especially conducive to the measurement of some complex environments. How to apply digital measurement in atmospheric environment monitoring is the focus of atmospheric environment monitoring [1]. Therefore, it is of great practical significance to explore the digital measurement strategy of atmospheric environment monitoring. In modern society, green environmental protection has become the theme of the times, and people begin to pay more attention to environmental issues. However, the gradual expansion of various production industries has had a certain impact on atmospheric environmental protection. This makes atmospheric environmental monitoring become a monitoring content that the relevant departments must pay attention to [2]. It is necessary to ensure its reasonable application, select appropriate layout methods, and ensure the accuracy of atmospheric environmental monitoring data [3].

Atmospheric environmental monitoring is a process of measuring the concentration of pollutants in the atmospheric environment, observing and analyzing their changes and their impact on the environment. Air pollution monitoring is to determine the types and concentrations of pollutants in the atmosphere and observe their temporal and spatial distribution and changes [4]. Usually, the pollutants in the monitoring area mainly include sulfur oxides, nitrogen oxides, carbon monoxide and so on, and the granular pollutants include falling dust, total suspended particulates, floating dust and acid deposition. With the development of the times, now the atmospheric environment is deteriorating, atmospheric change is easily affected by various factors, especially industrial production [5]. Through the rational application of atmospheric environmental monitoring, on the one hand, we can timely understand the concentration, type and range of various pollutants in the atmospheric environment, so as to obtain more basic data and compile atmospheric environmental quality reports [6]. On the

\footnotetext{
*Corresponding author: honghong03050305@163.com
} 
other hand, it is conducive to early warning, once largescale changes in pollutants in the atmospheric environment are found, an alarm can be made as soon as possible to avoid the possibility of air pollution to the greatest extent and provide adequate protection for people's health [7]. Combined with the actual situation of atmospheric environmental monitoring at the present stage, the specific application is mainly reflected in three aspects as shown in Figure 1.

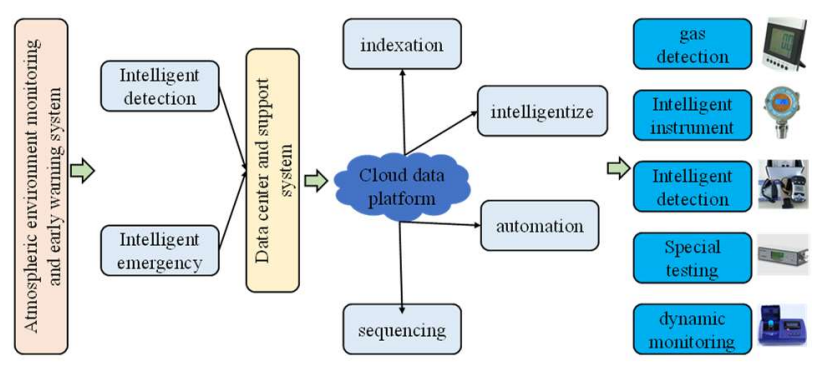

Figure 1 The method of Atmospheric Environment Detection

Based on the above analysis, it is not difficult to understand that atmospheric environmental monitoring is usually used in the monitoring of atmospheric pollutants, and provides necessary data reference for atmospheric research. The monitoring department must choose a reasonable monitoring method according to the characteristics of the environment to ensure the accuracy of the data to the maximum extent. In this paper, combined with the actual situation, the application and layout methods of atmospheric environmental monitoring are analyzed in detail, in order to provide reference and reference for the related work in the future. In this paper, it is pointed out that atmospheric environmental monitoring is the process of measuring the concentration, observation and analysis of the change of pollutants in the atmosphere and the shadow response of the environment. Digital monitoring technology is the product of the digital era, which has its own advantages in monitoring the atmospheric environment, such as climate assessment, environmental assessment, atmospheric disaster prevention and early warning and so on. Taking the digital measurement as the cut-in point, this paper describes the object and appearance of atmospheric monitoring, and focuses on the application strategy of digital measurement of atmospheric environment monitoring, in order to clarify the importance of digital measurement and provide reference.

\section{Test statistical method}

\subsection{Grid layout method}

Grid distribution is to divide the monitoring area into multiple grids, follow specific requirements and principles, and divide the grid evenly on the basis of ensuring reasonable division. In general, the setting of the sampling point should be at the center of the grid. If the situation is special, the sampling point will be set at the corner and position of the two straight lines of the grid, so as to ensure the representativeness of the sampling point. At the same time, in order to ensure the accuracy of atmospheric environmental monitoring, it is also necessary to determine the grid size, combined with the number of population, manpower and material conditions, the degree of pollution to make a comprehensive determination, a comprehensive analysis of atmospheric information, to determine the final sampling point. In general, the grid layout method is mainly applied to the rest of the serious pollution, or where the degree of pollution is uniform in the area, only in this case, can it meet the principle that "the location of the site should ensure that the pollution concentration is consistent with the surrounding concentration", and reflect the spatial information of pollution more truly and comprehensively.

\subsection{Fan layout method}

This method is similar to the concentric circle distribution method, in which a fan-shaped area is drawn in the downwind direction allowed by the regional condition and range, and the angle is set to $45^{\circ}$. According to the different conditions in the area, the angle can also be set to 60 degrees. No more than 90 degrees as far as possible. After the drawing is completed, the sampling points will be designed in fan-shaped arcs, with a number of 3-4. It should be noted that the angles of the two adjacent sampling points are controlled between 10 and 20 degrees, and the maximum cannot exceed 20 degrees. This layout method is mainly used in some areas where the dominant wind direction is more obvious, specific particularity, and the existence of relatively isolated high point sources, it can be said to be the most obvious difference among many methods. It is also the layout method with the most requirements and conditions, and the scope of application is too limited, so it needs specific analysis of specific problems to determine whether the sector layout method can be used.

\subsection{Functional area layout method}

Similar to the general statistical test method, as shown in Figure 2, this layout method is mainly based on the division of functional areas for layout and monitoring. Therefore, in the process of practical application, it is necessary to achieve accurate division of business, residential areas, industrial areas and so on, and this method can be chosen only when the conditions are available. However, affected by various factors, the division of these regional functions is often very difficult to achieve "fine". In order to change this situation, the relevant staff should have a detailed understanding of the regional population and pollution in the process of monitoring. In order to dynamically adjust the atmospheric environment monitoring work. If there are no special circumstances, in the process of monitoring, regular points can also be selected for monitoring, spoiler routine characteristics, the accuracy will be slightly reduced. Staff need to be fully aware of this and make a full study of each point when arranging points, so that a more representative location can be selected to ensure that 
each set of data is more accurate. In the process of practical application, this method requires that the pollution center point in the monitoring area must be determined, the concentric circle with different radius should be drawn around the center store, and the radiation should be done from different angles from the center of the circle. The position of the sampling point can be set at the focus of the ray. Affected by the monitoring environment, the number of sampling sites is not very stable, and the distribution is uneven. Generally, more sampling sites are arranged in the downwind direction of the dominant risk, and the upwind direction is relatively less.

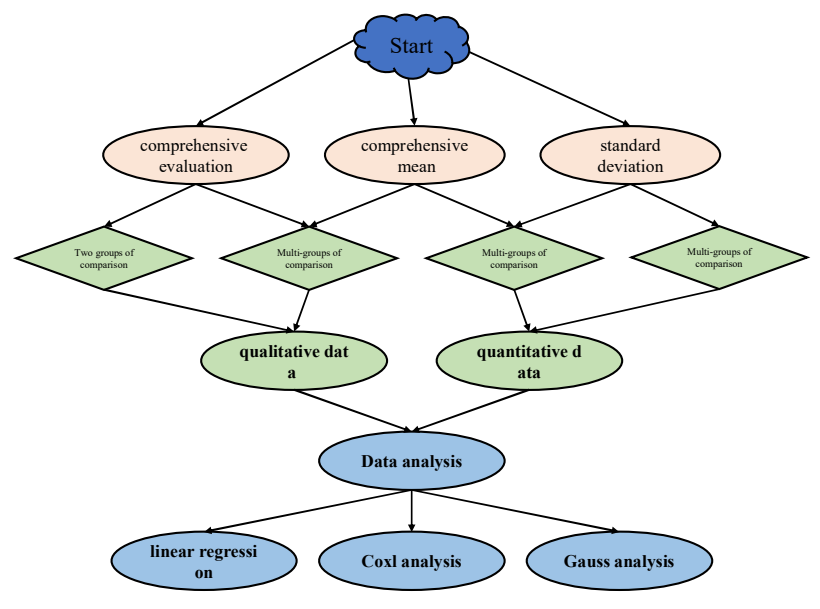

Figure 2 Statistical test method

\section{Effective way to optimize the layout of Atmospheric Environmental Monitoring}

\subsection{Reduce the scope of influence}

In the process of actual monitoring, the sampling points are likely to be affected by many factors and lead to judgment errors, which need to be further optimized to reduce the scope of influence as much as possible. At present, the commonly used optimization methods are as follows: first, the correlation coefficient method, in which the number of grids is set to $\mathrm{m}$, the number of monitoring points is $\mathrm{n}$, and the plot points of Shangfeng line are unlimited. When using the grid layout method, it is necessary to calculate the correlation coefficient $\mathrm{R}$ between $m$ and $n$, and then calculate the coefficient of variation and the variance of each point according to the regional pollution, the law of change and the average concentration, and then determine the location of the points after comprehensive consideration. Second, matter-element analysis requires that in the process of detection, the maximum and minimum values should be found, the average value should be calculated, and the average value should be combined with the highest value and the lowest value to form a standard matter-element matrix, from which the correlation function can be calculated. Draw the distribution point aggregation diagram to determine the best location of the layout point. Third, the testing method, after using the above method, we can divide and calculate the high, middle and low pollution areas according to the average concentration of pollutants in the region, determine the layout scheme according to the evaluation scheme, and choose the optimal layout method to ensure the rationality of the layout. Fourth, the optimal index method is a way and method based on the formation of TOPSIS. In the process of practical application, we should first establish the initial monitoring data matrix $\mathrm{M}$, get the optimal index box girder matrix $N$, unify $M$ to establish the final most decision-making matrix $Z$, calculate the difference between the monitoring point index and the most level, follow the order from big to small, and select the optimal location. Fifth, the characteristic analysis method, mainly according to the pollution concentration to achieve the division of the telephone, in which each category of beans should set up a representative distribution point, to meet the monitoring principle of the representative requirements of the distribution point, establish the correlation matrix and calculate, get the best location.

\subsection{Virtual measurement}

The digital measurement of atmospheric environment monitoring is inseparable from the application of virtual instrument and technology, the virtual instrument and computer technology are combined with each other, the high-performance modular hardware is used to measure the atmospheric environment, and the measured data can be used as the basis for atmospheric environment monitoring. It is more simple and convenient, and the accuracy is higher. Specifically, the panel of the virtual instrument is virtual, the main thing is to integrate the network communication technology and electronic technology, and the measurement function is mainly completed by software to realize the sharing of monitoring information. Among them, the panel of variability, multi-layer and self-creation in the virtual instrument can better configure the atmospheric data resources, calculate, analyze and judge the atmospheric environment monitoring data flexibly, and realize the interconnection between the virtual instrument and the peripheral equipment. The automatic test system with virtual instrument technology as the trend is also conducive to the realization of real-time monitoring of atmospheric environment and has more practical application value. For example, the application of excited light radar is very effective in detecting atmospheric components and their pollutants. Lidar can detect the specific distribution of air-dissolved limbs and cloud particles, and at the same time, it can detect large gas components and pollute environmental gases, so as to obtain high-precision data and improve the quality of atmospheric data.

As the application front end of digital measurement, the application of digital server function just realizes the human-computer interaction. The function of the data server is a comprehensive system support. According to the sampling, storage and analysis of the atmospheric environment monitoring data, and the sampling server recording the atmospheric environment monitoring data, 
it should be noted that the sampling work seems simple, but in fact it is not, although it is the most basic work of atmospheric environment monitoring. However, it is also the most critical part, and whether the sampling data is accurate or not directly affects the results of atmospheric environment monitoring. The selection of sampling points and the time of sampling are very important. First of all, the sampling site must be representative, that is to say, the sampling site should represent the general pollution level of the area and reflect the pollution situation of the atmospheric environment in the region. Secondly, the sampling time should be marked and marked at the right time to ensure that the number of samples collected is true and useful. After the networking, the data is supported and finally stored in the data database for use. Here, it involves the storage of atmospheric environmental monitoring data, and the storage function can store the data tested in the past. The data of atmospheric environmental monitoring are more intuitive and clear, which lays a foundation for the analysis of atmospheric environmental monitoring data. Not only that, but in the application of the data server, manual operation can also be carried out. When necessary, according to the needs of the atmospheric monitoring and measurement work, the manual input modifies the parameter setting, the management of the data is more convenient, and the man-machine interaction is coordinated and cooperated with each other. The amount of work in data processing is reduced. So that the atmospheric environment control methods and control hands are more optimized. For example, in the process of investigating air pollution, a large number of real-time monitored data can be collected by carrying the gas sensor on the UAV.

\section{Atmospheric environment assessment}

\subsection{Collect accurate environmental data}

Ensuring the accuracy of basic data is the basic requirement of atmospheric environmental assessment. In addition to conventional data, such as meteorological data, topographic data, pollution data, etc., we should also focus on the detection data of the current regional situation, changes in the distribution of regional pollution sources, project plans and environmental management materials. The detection data of the current situation of the area can directly reflect the change degree of air quality in this area as shown in Figure 3, and the distribution change materials of regional pollution sources can provide reference for the change of the number of pollution sources in each stage of the implementation of the planning project. The feasibility of the planning project can be effectively judged by analyzing the project proposal through environmental management materials.

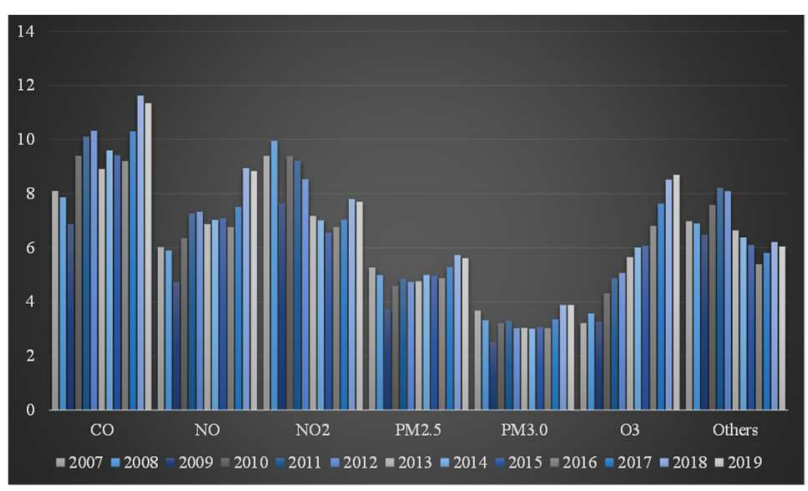

Figure 3 Collect and collate environmental data

\subsection{Develop a prediction scheme for environmental impact}

The environmental impact prediction scheme is carried out through the selection of pollution sources and emission locations in different planning periods, including simulating environmental quality changes, short-term environmental impact prediction, long-term environmental impact prediction and so on. In order to reduce the workload and improve work efficiency. Among them, it is necessary to accurately analyze the air pollution sources and understand the type, discharge and distribution of the pollution sources. In the process of construction, the emissions, discharge volume and location are uncertain, so it is impossible to carry out accurate quantitative analysis. Therefore, it is necessary to use effective methods to carry out analysis, such as analogy analysis, to compare the contents involved in the planning project with the relevant data in the industry, and to analyze the comparison of scale, process and output. As a result, the quantity, category and nature of pollutants can be predicted as shown in Figure 4. Coefficient method, this method is based on the database constructed by data analysis in the industry, and the prediction results can be obtained according to the emission coefficient. Finally, the prediction scheme also needs to determine the evaluation factors, calculation points and prediction areas, so that the workload can be reduced. Detailed analysis of the planning plan, with reference to the local meteorological characteristics, local leading industries and combined with the latest environmental management policy to analyze the content of the planning scheme to judge the maneuverability of the plan. If a certain area is planned, it is necessary to select the atmospheric index and formulate the planning plan according to the regional characteristics. China's environmental control quality standards have put forward clear indicators for various types of pollutants, which also need to be referred to when implementing the planning plan. In addition, it is necessary to compare and select the planning schemes according to the results of the prediction and evaluation, according to the regional concentration and key points mentioned in the planning scheme, and synthesize the factors such as location, scale, layout and industrial structure, and finally get the adjustment opinion. According to the characteristics of different planning projects, optimization suggestions are put forward mainly 
from three aspects: site layout, scale and industry, and analysis results. The optimization of the scheme is carried out according to the analysis results. If the deviation between the project analysis results and the reality is too large, the planning scheme should be cancelled to avoid economic losses.

a
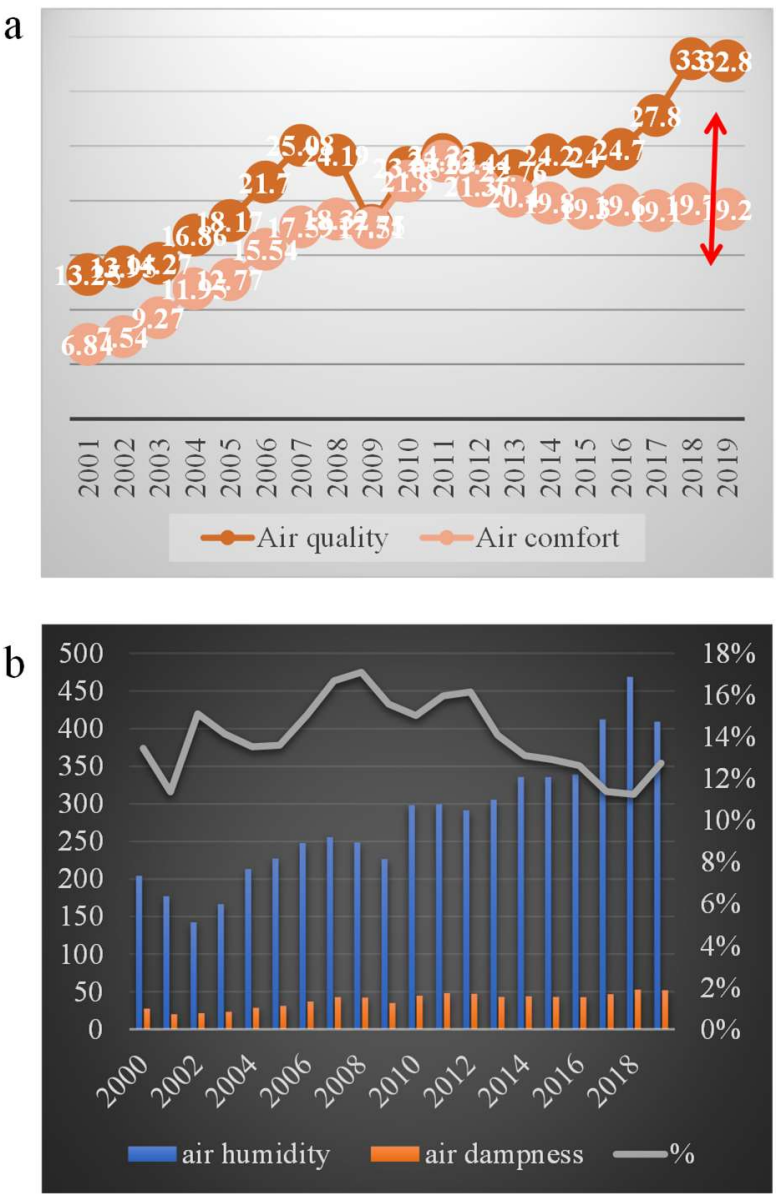

Figure 4 Effect of polymerization degree

\section{Conclusion}

Atmospheric environmental monitoring is usually used in the monitoring of atmospheric pollutants and provides necessary data reference for atmospheric research. The monitoring department must choose a reasonable monitoring method according to the characteristics of the environment to ensure the accuracy of the data to the maximum extent. In this paper, combined with the actual situation, the application and layout method of atmospheric environment monitoring will be analyzed in detail by using the method of experimental statistics, in order to provide reference and reference for the related work in the future. In this paper, it is pointed out that atmospheric environmental monitoring is the process of measuring the concentration, observation and analysis of the change of pollutants in the atmosphere and the shadow response of the environment. Digital monitoring technology is the product of the digital era, which has its own advantages in monitoring the atmospheric environment, such as climate assessment, environmental assessment, atmospheric disaster prevention and early warning and so on. Taking the digital measurement as the cut-in point, this paper describes the object and appearance of atmospheric monitoring, and focuses on the application strategy of digital measurement of atmospheric environment monitoring, in order to clarify the importance of digital measurement and provide reference.

\section{Acknowledgements}

If any, should be placed before the references section without numbering.

\section{References}

1. Hunter, James F., et al. "Comprehensive Characterization of Atmospheric Organic Carbon at a Forested Site." Nature Geoscience, vol. 10, no. 10, pp. 748-753, 2017.

2. Ochoa-Hueso, Raúl, et al. "Ecological Impacts of Atmospheric Pollution and Interactions with Climate Change in Terrestrial Ecosystems of the Mediterranean Basin: Current Research and Future Directions." Environmental Pollution, vol. 227, pp. 194-206, 2017.

3. Yu, Haili, et al. "Development of Atmospheric Acid Deposition in China from the 1990s to the 2010s." Environmental Pollution, vol. 231, pp. 182-190, 2017.

4. Ramsay, David B., et al. "Making Air (Quality) Visible: Exploiting New Technology to Dramatically Improve Atmospheric Monitoring." IEEE Pervasive Computing, vol. 17, no. 3, pp. 90-94, 2018.

5. Hofman, Jelle, et al. "Biomagnetic Monitoring of Atmospheric Pollution: A Review of Magnetic Signatures from Biological Sensors." Environmental Science \& Technology, vol. 51, no. 12, pp. 66486664, 2017.

6. Chen, Long, et al. "Atmospheric Mercury Outflow from China and Interprovincial Trade." Environmental Science \& Technology, vol. 52, no. 23, pp. 13792-13800, 2018.

7. Bazarov, A. V., et al. "Mobile Measurement System for the Coupled Monitoring of Atmospheric and Soil Parameters." Russian Meteorology and Hydrology, vol. 43, no. 4, pp. 271-275, 2018. 